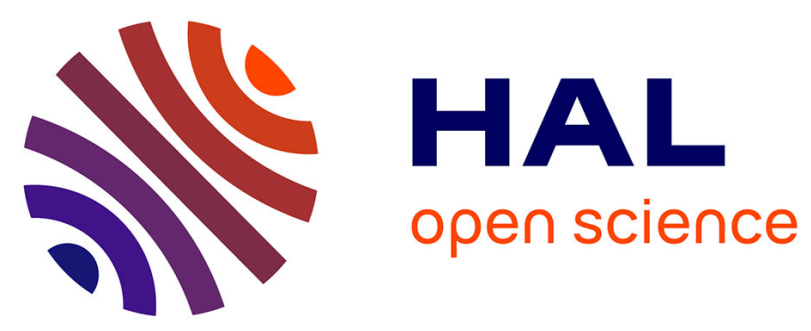

\title{
Reconstruction of heterogeneous surface residual-stresses in metallic materials from X-ray diffraction measurements
}

Léo Morin, Chedly Braham, Pouya Tajdary, Raoudha Seddik, Gonzalo Gonzalez

\section{To cite this version:}

Léo Morin, Chedly Braham, Pouya Tajdary, Raoudha Seddik, Gonzalo Gonzalez. Reconstruction of heterogeneous surface residual-stresses in metallic materials from X-ray diffraction measurements. Mechanics of Materials, 2021, 158, pp.103882. 10.1016/j.mechmat.2021.103882 . hal-03218418

\section{HAL Id: hal-03218418 https://hal.science/hal-03218418}

Submitted on 5 May 2021

HAL is a multi-disciplinary open access archive for the deposit and dissemination of scientific research documents, whether they are published or not. The documents may come from teaching and research institutions in France or abroad, or from public or private research centers.
L'archive ouverte pluridisciplinaire HAL, est destinée au dépôt et à la diffusion de documents scientifiques de niveau recherche, publiés ou non, émanant des établissements d'enseignement et de recherche français ou étrangers, des laboratoires publics ou privés. 


\title{
Reconstruction of heterogeneous surface residual-stresses in metallic materials from X-ray diffraction measurements
}

\author{
Léo Morin $^{\mathrm{a}, *}$, Chedly Braham ${ }^{\mathrm{a}}$, Pouya Tajdary ${ }^{\mathrm{a}}$, Raoudha Seddik ${ }^{\mathrm{a}}$, Gonzalo Gonzalez ${ }^{\mathrm{b}}$ \\ a PIMM, Arts et Metiers Institute of Technology, CNRS, Cnam, HESAM University, 151 boulevard de l'Hopital, 75013 Paris, France \\ ${ }^{\mathrm{b}}$ Instituto de Investigaciones en Materiales, Universidad Nacional Autónoma de México, Circuito Exterior S/N, Cd. Universitaria, A.P. 70-360, \\ Coyoacán, 04360 México, D.F., Mexico
}

Keywords:

$\mathrm{X}$-ray diffraction

Residual stresses

Aluminum alloys

Severe plastic deformation

Laser shock peening

\begin{abstract}
A B S T R A C T
The aim of this paper is to provide spatially resolved distributions of residual stresses. X-ray diffraction measurements provide an intrinsic average of the residual stress due to the diffracted volume analyzed during the measurement. When the irradiated area is higher than the characteristic length of stress gradients, strong averaging effects are observed. A spatial deconvolution technique is developed to reconstruct the local residual stress field, based on the inversion of a linear system constructed from the average datasets. The method is first applied to the reconstruction of residual stresses in two reference cases inducing heterogeneous plastic strains (laser shot peening and repetitive corrugation and straightening processing), in which the average datasets are constructed from the local stress profiles determined numerically by the finite element method. In both processes, a very good agreement is observed between the reference stress profiles and the reconstructed ones. Finally, the method is applied to experimental X-ray diffraction measurements on a specimen processed by repetitive corrugation and straightening in similar conditions than the numerical simulations. A strong averaging effect is observed on the collected data and a good agreement is observed between the local stress profile reconstructed from the experimental measurements and that predicted numerically.
\end{abstract}

\section{Introduction}

Residual stresses have a significant influence on the engineering properties of materials and structural components such as fatigue life, corrosion resistance and resistance to brittle fracture. They constitute one of the major factors determining the structural integrity of engineering components and the control of its underlying mechanisms is of great interest during the design and manufacturing of products (Webster and Ezeilo, 2001; Vaara et al., 2020). Residual stresses are due to spatial gradients of irreversible strains which typically originate from heterogeneous plastic deformation or phase transition. Thus, residual stresses can be generated unintentionally during most manufacturing processes involving irreversible deformation and heat treatment such as in machining (Jacobus et al., 2000; Zhang et al., 2016; Ben Rhouma et al., 2019), welding (Dong and Brust, 2000; Leggatt, 2008), laser marking (Lu et al., 2020), severe plastic deformation (Reyes-Ruiz et al., 2016; Ezequiel et al., 2020) and additive manufacturing (Fang et al., 2020); the presence of undesirable tensile residual stresses can decrease fatigue life and corrosion resistance. On the other hand, compressive residual stresses can be induced on purpose in order to improve durability, by means of several processes such as shot peening (Hammond and Meguid, 1990; Mahmoudi et al., 2016), laser shock peening (Peyre and Fabbro, 1995; Montross et al., 2002) and wire brushing (Ben Fredj et al., 2004). The assessment and prediction of residual stress distributions in engineering components is thus a major challenge for durability and reliability.

In terms of experimental measurement techniques, significant advances have been made to provide accurate distributions of residual stresses (Rossini et al., 2012), based on several experimental methods including X-ray diffraction, neutron diffraction, deep hole drilling and magnetic methods, among others. X-ray diffraction (XRD) constitutes a high accuracy and non-destructive way to determine residual stresses in crystalline materials, where crystalline planes are used as strain gauges ( $\mathrm{Lu}, 1996)$. Based on the knowledge of the wavelength and the measured shift of the Bragg angle, the change in interatomic lattice spacing can be calculated, which permits to evaluate the strain in a small volume under the specimen surface. Residual stresses can then be deduced from the elastic theory provided that the X-ray elastic constants are known. Due to the important absorption of X-ray in metallic alloys, the method allows measurements in outer layers. Thus, in conjunction with successive polishing, the XRD method permits 
to determine (destructively) stress gradients in depth with a very good precision and resolution. Advanced techniques based on multireflection grazing-incidence allow to provide depth-dependent stress profiles non-destructively (Baczmanski et al., 2004; Marciszko et al., 2017).

Despite its important success and its high precision in-depth, the $\mathrm{X}$-ray diffraction method remains inaccurate in the presence of high surface stress gradients (Kahloun et al., 1994; Hennion et al., 2000; Kahloun et al., 2014). Indeed, when the surface gradient of residual stresses is smaller than the irradiated zone, the stresses obtained from XRD correspond to some average of the local stresses because X-ray diffraction only yields the mean lattice strains over the irradiated area (whose size will depend on the collimator geometry). A decrease of the irradiated zone permits to improve the homogeneity of the surface stress, but, since the diffracting volume has to contain a sufficient number of crystallites to be statistically representative, the irradiated area cannot be reduced below some critical value in typical engineering materials containing micron-sized crystallites. Thus in the presence of high lateral stress gradients, inherent averaging effects are expected to occur on the stresses measured by X-ray diffraction.

The quality of the local residual stress profiles obtained experimentally by X-ray diffraction can thus be very questionable in the zones having high lateral stress gradients, which raises important issues notably in processing validation or for the determination of threshold criteria in stress corrosion cracking (Ben Rhouma et al., 2019). In particular, in the context of process simulation, the averaging effects on residual stresses measured by X-ray diffraction prevent a proper comparison with the local residual stresses determined numerically by the finite element method. For instance, this effect may explain the discrepancies observed notably in the comparison between experiments and numerical stress profiles in heterogeneous processing such as laser shock peening (Peyre et al., 2003, 2012), in contrast with (more) homogeneous processes like classical shot peening (Xie et al., 2016; Zhou et al., 2018). It is worth noting that this important drawback of the X-ray diffraction method has only been studied by a few authors:

- A first way to circumvent the averaging issue is to post-treat the finite element results by making an average of the residual stress field over the XRD irradiated area. This was investigated in the case of a multi-impact laser shock peening processing (Brockman et al., 2012). Although this method is interesting because it accounts for the X-ray averaging effects in the finite element simulations, it does not permit to capture the experimental heterogeneities as it only gives information on the averaged stresses.

- A second way was proposed through the deconvolution of the measured peak parameter, allowing a correction of the residual stress field accounting for the irradiated area and penumbra effect (Hennion et al., 2000). However, since the method is based on the peak parameter deconvolution, it can provide a reconstructed stress profile only for a given irradiated area. Thus, when applied to the reconstruction of the stress profile in a melted specimen in two experimental conditions with two irradiated areas, the method proposed by Hennion et al. (2000) has given two reconstructed stress profiles (for each irradiated areas) which were very different, emphasizing that their method does not permit to reconstruct a unique and precise local stress profile.

- Mention has to be made to the work of Kahloun et al. (2014), who investigated the problem of stress deconvolution in XRD measurement. However, since this method makes use of Fourier transforms of the convoluted stress, it restricts the residual stress distributions to periodic functions. Furthermore, their inverse procedure is ill-posed due to the experimental noise and the lack of redundant data, so the method does not appear suitable to reconstruct accurate local stress distributions.
An alternative and new approach is considered in this work. It consists in the spatial reconstruction of the local residual stress field from several X-ray diffraction measurements performed on a regular grid (e.g. using an automatic robot) and with the use of several collimator sizes inducing various irradiated areas. Indeed, since the average stress profile obtained by XRD consists in the convolution of the stress field over the irradiated area (Kahloun et al., 2014), it is possible to establish a linear relationship between the point-wise values of the local stress field and that of the average stress determined experimentally. The combination of a fine measurement grid and several collimator sizes allows the construction of an overdetermined linear system. Provided that the average stresses are smoothed to decrease experimental noise, the approximate inversion of the overdetermined system (using ordinary least squares method) should then lead to a deconvolution and reconstruction of a unique and precise local stress field.

The paper is organized as follows. In Section 2, a deconvolution method, based on the inversion of a linear system depending on the average datasets, is developed to reconstruct spatially the local stress. The method is applied in Section 3 to the reconstruction of local residual stresses in two reference cases inducing heterogeneous strains (laser shock peening and repetitive corrugation and straightening processing) which are computed numerically. Finally, the spatial stress deconvolution method is applied to experimental data collected on a specimen processed by severe plastic deformation.

\section{A deconvolution method of spatial residual-stresses using X-ray diffraction measurements}

\subsection{Averaging effects in X-ray diffraction measurements}

The stresses obtained from XRD measurements result from a convolution of the local stress over the irradiated area, which is generally of circular or rectangle shape in practical laboratory facilities (see Fig. 1a). Throughout this paper we will assume the following hypotheses, which will allow the derivation of a stress deconvolution method that can be used in practical applications:

(1) The irradiated area is square-shaped;

(2) The lateral stress-gradient occurs in one direction;

(3) The averaging over the irradiated area is uniform (no penumbra effects).

Thus under these hypotheses, the problem considered is onedimensional so the XRD experiments can be performed on a line. We assume that the local residual-stress field is a continuous function $\sigma(x)$, where $x$ is the spatial coordinates defined in the domain $[0, L]$. X-ray diffraction measurements permit to obtain some average value of the stress field $\sigma(x)$ over a moving interval of width $2 a$, corresponding to the irradiated diameter (see Fig. 1b).

The moving average of the stress field $\sigma(x)$, which is denoted by $\Sigma^{a}(x)$, is given by the formula

$\Sigma^{a}(x)=\frac{1}{2 a} \int_{x-a}^{x+a} \sigma(u) \mathrm{d} u$.

In practice, the moving average $\Sigma^{a}$ is restricted to a finite number of experimental points. The interval of definition of the residual-stress field $\sigma,[0, L]$, is discretized into uniform grid with spatial scale $\Delta x=$ $L / N$, where the total number of points is $N+1$. We use the following notation: $x_{i}=i \Delta x, \sigma_{i}=\sigma\left(x_{i}\right)$, with $i=0, \ldots, N$. For simplicity, we assume that the diameter of the irradiated zone is supposed to coincide with the grid, which implies that $a$ is of the form

$a=k_{a} \Delta x$,

where $k_{a}$ is a positive integer. Since the moving average is defined in the interval $[a, L-a]$, its discrete values $\Sigma_{i}^{a}=\Sigma^{a}\left(x_{i}\right)$ are defined with $i=k_{a}, \ldots, N-k_{a}$ and may thus be written as a vector $\boldsymbol{\Sigma}^{a}$ of size $N+1-2 k_{a}$. We are thus looking for the discrete values $\sigma_{i}$ of the local stress field at the points $x_{i}$, which consists in finding the vector $\sigma$ of size $N+1$. 

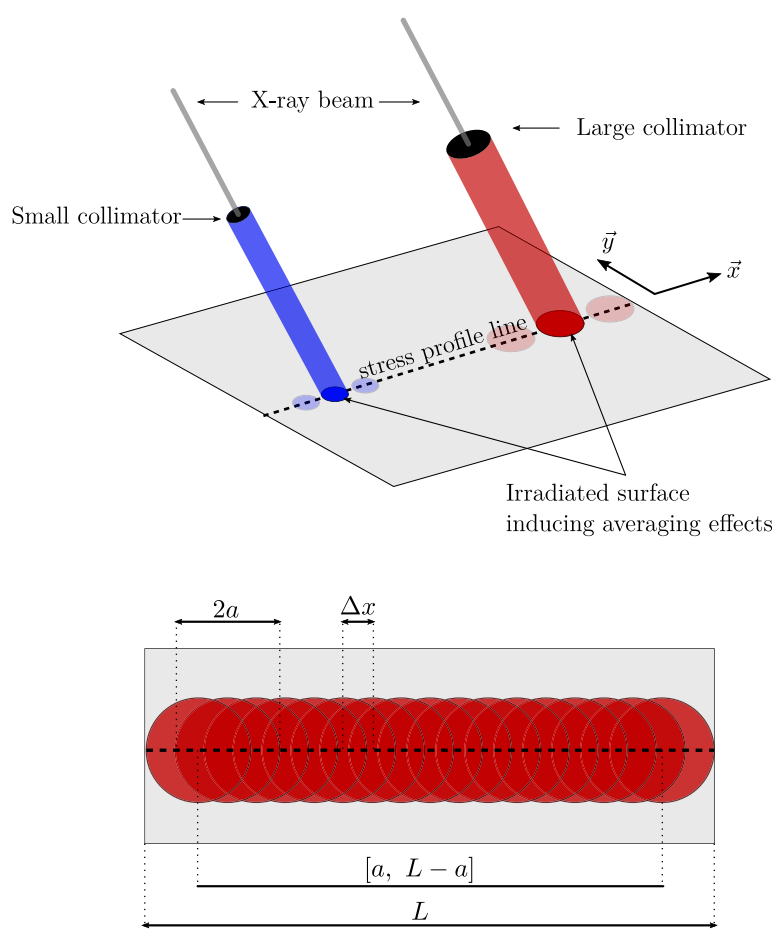

(a)

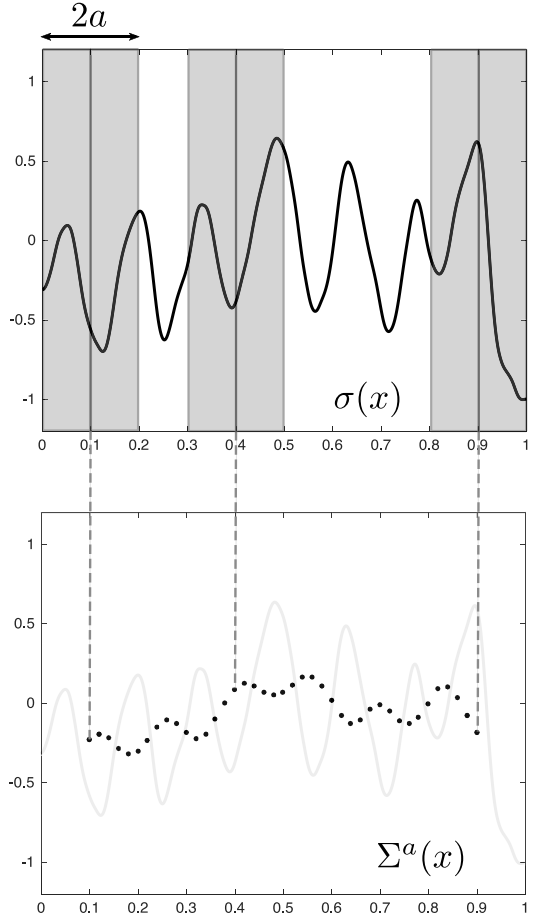

(b)

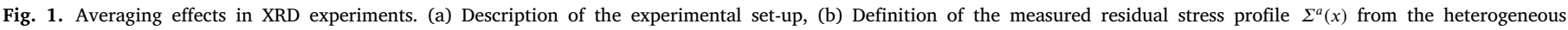
residual stress profile $\sigma(x)$.

\subsection{Deconvolution by integration approximation}

The most natural approach for computing the vector $\sigma$ from its moving average $\boldsymbol{\Sigma}^{a}$ is to approximate the integral in Eq. (1), using a trapezoidal rule. At the grid point $x_{i}$, it reads:

$\Sigma_{i}^{k_{a}}=\frac{1}{2 k_{a}}\left(\frac{\sigma_{i-k_{a}}+\sigma_{i+k_{a}}}{2}+\sum_{j=1}^{j=2 k_{a}-1} \sigma_{i-k_{a}+j}\right), \quad i=k_{a}, \ldots, N-k_{a}$.

The $\left(N+1-2 k_{a}\right)$ equations defined by (3) lead to the linear system

$\boldsymbol{\Sigma}^{a}=\mathbf{R}^{a} \boldsymbol{\sigma}$

where $\mathbf{R}^{a}$ is a $\left(N+1-2 k_{a}\right) \times(N+1)$ matrix given by

$\mathbf{R}^{a}=\left(\begin{array}{ccccccccc}r_{0}^{a} & r_{1}^{a} & \ldots & r_{2 k_{a}-1}^{a} & r_{2 k_{a}}^{a} & 0 & 0 & \ldots & 0 \\ 0 & r_{0}^{a} & r_{1}^{a} & \ldots & r_{2 k_{a}-1}^{a} & r_{2 k_{a}}^{a} & 0 & \ldots & 0 \\ \vdots & & \ddots & \ddots & & \ddots & \ddots & & \vdots \\ 0 & \ldots & 0 & r_{0}^{a} & r_{1}^{a} & \ldots & r_{2 k_{a}-1}^{a} & r_{2 k_{a}}^{a} & 0 \\ 0 & \ldots & 0 & 0 & r_{0}^{a} & r_{1}^{a} & \ldots & r_{2 k_{a}-1}^{a} & r_{2 k_{a}}^{a}\end{array}\right)$.

The non-zero components of the matrix $\mathbf{R}^{a}$ are given by

$r_{i}^{a}= \begin{cases}\frac{1}{2 k_{a}} & \text { if } 1 \leq i \leq 2 k_{a}-1, \\ \frac{1}{4 k_{a}} & \text { if } i=0 \\ \frac{1}{4 k_{a}} & \text { if } i=2 k_{a} .\end{cases}$

The linear system (4) is underdetermined since there are fewer equations $\left(N+1-2 k_{a}\right)$ than unknowns $(N+1)$. In that case, the classical method of ordinary least squares can be used to find an approximate solution of the system, which consists in minimizing the residual sum-of-squares defined as

$\left\|\mathbf{R}^{a} \sigma-\Sigma^{a}\right\|$, where $\|\cdot\|$ denotes the (classical) Euclidean norm. The solution of this minimization problem leads to

$\boldsymbol{\sigma}=\left(\mathbf{R}^{a}\right)^{+} \boldsymbol{\Sigma}^{a}$,

where $\left(\mathbf{R}^{a}\right)^{+}$is the Moore-Penrose right pseudoinverse given by

$\left(\mathbf{R}^{a}\right)^{+}=\left(\mathbf{R}^{a}\right)^{T}\left(\mathbf{R}^{a}\left(\mathbf{R}^{a}\right)^{T}\right)^{-1}$.

The quality of the approximate solution field $\sigma$ given by Eq. (8) is expected to be poor because (i) the system is undetermined and (ii) experimental noise is expected on $\boldsymbol{\Sigma}^{a}$ (Engl et al., 1996). Remedies are thus needed to provide a satisfactory reconstruction.

\subsection{Automatic spline smoothing of noisy data}

In practice, the moving average vector is determined up to measurement errors. This has important consequences upon the inverse problem considered since it may generate important spurious oscillations (Engl et al., 1996). In this context, an efficient way to remove the experimental noise, by separating it from the dataset, consists in using advanced smoothing techniques.

In the case of non-periodic data, smoothing splines are a very effective method to construct of a smooth estimate $\widetilde{\boldsymbol{\Sigma}}^{a}$ of $\boldsymbol{\Sigma}^{a}$ by minimization of a functional $\mathcal{G}$ that balances the fidelity to the data, through the residual sum-of-squares (RSS), and the smoothness of the estimate $\widetilde{\boldsymbol{\Sigma}}^{a}$, through some penalty term $\mathcal{P}$ (Garcia, 2010):

$\mathcal{G}\left(\widetilde{\boldsymbol{\Sigma}}^{a}\right)=\left\|\boldsymbol{\Sigma}^{a}-\widetilde{\boldsymbol{\Sigma}}^{a}\right\|^{2}+s \mathcal{P}\left(\widetilde{\boldsymbol{\Sigma}}^{a}\right)$,

where $\|\cdot\|$ denotes the Euclidean norm and $s$ is a real positive scalar that controls the degree of smoothing. The penalty term is expressed in terms of the point-values of the $p$ th derivative of $\widetilde{\boldsymbol{\Sigma}}^{a}$ at the grid points. In the case of the second-order derivative, it reads

$\mathcal{P}\left(\widetilde{\boldsymbol{\Sigma}}^{a}\right)=\left\|\mathbf{D} \widetilde{\boldsymbol{\Sigma}}^{a}\right\|^{2}$ 
where $\mathbf{D}$ is the second-order differentiation matrix given by

$\mathbf{D}=\frac{1}{\Delta x^{2}}\left(\begin{array}{ccccc}-1 & 1 & & \ldots & 0 \\ 1 & -2 & 1 & & \vdots \\ 0 & \ddots & \ddots & \ddots & 0 \\ \vdots & & 1 & -2 & 1 \\ 0 & \ldots & & 1 & -1\end{array}\right)$.

The minimization of $\mathcal{G}$ leads to the expression of the smooth estimate $\tilde{\boldsymbol{\Sigma}}^{a}$ (Garcia, 2010)

$\tilde{\boldsymbol{\Sigma}}^{a}=\operatorname{IDCT}\left(\boldsymbol{\Gamma}(s) \circ \operatorname{DCT}\left(\boldsymbol{\Sigma}^{a}\right)\right)$,

where DCT and IDCT respectively refer to the discrete cosine transform and the inverse cosine transform, and o denotes Hadamard product (pointwise product). $\Gamma(s)$ is a vector of size $N+1-2 k_{a}$ (which controls the smoothness through the value of parameter $s$ ), whose components are given by

$\Gamma_{i}=\frac{1}{1+s \lambda_{i}^{2}}$,

where the parameter $\lambda_{i}$ is given by

$\lambda_{i}=2-2 \cos \left(\frac{(i-1) \pi}{N+1-2 k_{a}}\right)$.

The last step is to estimate the best smoothing parameter $s$ that avoids over- or under-smoothing as much as possible. Such estimation can be done using the classical method of generalized cross-validation (GCV) introduced by Craven and Wahba (1978), which consists in minimizing the so-called GCV-score. In the case of the smoother given by Eq. (13), the GCV score is given by

$\operatorname{GCV}(s)=\frac{\left(N+1-2 k_{a}\right) \sum_{i=1}^{N+1-2 k_{a}}\left(\frac{1}{1+s \lambda_{i}^{2}}-1\right)^{2} \operatorname{DCT}_{i}^{2}\left(\Sigma^{a}\right)}{\left(\left(N+1-2 k_{a}\right)-\sum_{i=1}^{N+1-2 k_{a}} \frac{1}{1+s \lambda_{i}^{2}}\right)^{2}}$.

In practice, the minimization in Eq. (16) can be easily performed using efficient routines based on the simplex method.

The smooth of the experimental data is thus fully automatic and the smoothing parameter estimate provided by the GCV method is unique (Garcia, 2010, 2011).

\subsection{Improved accuracy using several sets of measurements}

The method of deconvolution by integration approximation expounded in Section 2.2 is based on the approximate inversion of an undetermined system. There are fewer equations than unknowns which is not suitable to make a precise reconstruction. In order to improve the accuracy of the reconstruction, it is proposed to increase the number of experimental data by considering several sets of moving average. In practice, this can be easily achieved by using different collimators (and thus different irradiated areas) which would provide different moving average functions. Thus, we consider $n$ sets of measures associated with width denoted by $2 a_{j}(j=1, \ldots, n)$ and the moving average functions are defined such as

$\Sigma^{a_{j}}(x)=\frac{1}{2 a_{j}} \int_{x-a_{j}}^{x+a_{j}} \sigma(u) \mathrm{d} u$.

This allows the definition of a linear system involving

$p=\sum_{i=1}^{n}\left(N+1-2 k_{a_{i}}\right)$

equations arising from the definition of the moving average functions $\boldsymbol{\Sigma}^{a_{j}}$ for each set of irradiated width $2 a_{j}(j=1, \ldots, n)$. This linear system can be formally written as

$\boldsymbol{\Sigma}=\mathbf{R} \boldsymbol{\sigma}$, where $\boldsymbol{\sigma}$ is the vector of unknowns (of size $N+1$ ). In Eq. (19), $\boldsymbol{\Sigma}$ is a vector of size $p$ which concatenates the vectors $\widetilde{\Sigma}^{a_{j}}$ (obtained after the smoothing of $\left.\boldsymbol{\Sigma}^{a_{j}}\right)$, and $\mathbf{R}$ is a matrix of size $p \times(N+1)$ which concatenates the matrices $\mathbf{R}^{a_{j}}$ :



$\left.\mathbf{R}=\left(\begin{array}{ccccccccc}r_{0}^{a_{1}} & r_{1}^{a_{1}} & \ldots & r_{2 k_{a_{1}-1}}^{a_{1}} & r_{2 k_{a_{1}}}^{a_{1}} & 0 & 0 & \ldots & 0 \\ 0 & r_{0}^{a_{1}} & r_{1}^{a_{1}} & \ldots & r_{2 k_{a_{1}-1}}^{a_{1}} & r_{2 k_{a_{1}}}^{a_{1}} & 0 & \ldots & 0 \\ \vdots & & \ddots & \ddots & r_{1} & \ldots & \ddots & & \vdots \\ 0 & \ldots & 0 & r_{0}^{a_{1}} & r_{1}^{a_{1}} & \ldots & r_{2 k_{a_{1}-1}}^{a_{1}} & r_{2 k_{a_{1}}}^{a_{1}} & 0 \\ 0 & \ldots & 0 & 0 & r_{0}^{a_{1}} & r_{1}^{a_{1}} & \ldots & r_{2 k_{a_{1}-1}}^{a_{1}} & r_{2 k_{a_{1}}}^{a_{1}}\end{array}\right]\right)$

This system becomes overdetermined when $p \geq N+1$. Like in the previous case of an underdetermined system, the classical method of ordinary least squares can be used to find an approximate and unique solution of the system, which consists in minimizing the residual sum-of-squares defined as

$\|\mathbf{R} \sigma-\boldsymbol{\Sigma}\|$

The approximate solution of the minimization problem reads

$\boldsymbol{\sigma}=\mathbf{R}^{+} \boldsymbol{\Sigma}$

where $\mathbf{R}^{+}$is the Moore-Penrose left pseudoinverse given by

$\mathbf{R}^{+}=\left(\mathbf{R}^{T} \mathbf{R}\right)^{-1} \mathbf{R}^{T}$

The deconvolution method given by Eq. (22) thus provides a unique reconstructed local residual stresses from several average datasets collected from X-ray diffraction data. 


\subsection{Practical implementation of the method}

The deconvolution method has the advantage of reconstructing a unique local residual stress at the grid points where the X-ray diffraction measurements of the stress averages are performed. Despite its simplicity, several precautions, related to the practical implementation of the method, are needed in order to ensure the precision of the reconstructed residual stress. The following steps should then be followed:

(1) Select several collimators with different sizes. The use of several collimators will highlight the (possible) averaging effects of the measure.

(2) Adjust the spatial step. When the collimator sizes are selected, it is then necessary to adjust the spatial step $\Delta x$. Indeed, since in the deconvolution method, the moving average is calculated from the local stress by a trapezoidal rule, it is necessary to have a minimum number of points to calculate accurately the approximate integral.

(3) Perform XRD measurements at each point of the grid. Once the spatial step is known, the use of an automatic robot is required to perform automatically the XRD measurements at the grid points with a sufficient precision.

(4) Reconstruct the local stress. The acquired stress averages are smoothed using the automatic spline smoother and the local stress $\sigma$ is deduced using Eq. (22). The solution stress should be carefully checked because important (and non-physical) oscillations can result from the inversion. In such case, the automatic spline smoother should also be used on the reconstructed stress to remove these oscillations.

\section{Application of the method to simulation results}

The aim of this section is to assess the deconvolution method in reference cases where the local residual stress field is known. The deconvolution method will thus be applied to the reconstruction of residual stresses determined numerically by the finite element method; the moving average will be calculated from the local (known) residual stress profiles. Several datasets of moving averages, including additional noise mimicking measurement uncertainties, will be generated to assess the efficiency of the method. In order to assess solely the reconstruction method, the average stress field will be computed by considering (i) only the gradient in the direction of the mapping and (ii) no penumbra effects.

The method will be investigated in two very different processes that concern engineering materials, which are known to be both intrinsically heterogeneous: (i) laser shock peening (LSP) which induces intentional residual stresses and (ii) repetitive corrugation and straightening (RCS), belonging to severe plastic deformation processing, which also induces residual stresses. Even though the heterogeneity of the mechanical fields can be decreased by considering overlapping in LSP and a repetition of passes in RCS, the homogeneity of residual stresses in surface cannot be completely reached in both processes so it is of high interest to assess the fluctuation of residual stresses in high-gradient zones.

\subsection{Reconstruction of residual stresses in laser shock peening processing}

We begin with the numerical simulation of residual stresses in laser shock peening (LSP) processing. This technique is based on the blowoff of a very high-pressure plasma due to the laser-matter interaction, resulting in the propagation of mechanical waves in the specimen treated, inducing compressive residual stresses which permits to enhance fatigue properties (Peyre and Fabbro, 1995; Montross et al., 2002).
Table 1

Material properties and Johnson-Cook parameters for aluminum alloy AL000645 used for laser shock peening simulation.

\begin{tabular}{llllllll}
\hline Material & $E(\mathrm{GPa})$ & $v$ & $\sigma_{0}(\mathrm{MPa})$ & $h(\mathrm{MPa})$ & $n$ & $C$ & $\epsilon_{0}$ \\
\hline AA AL000645 & 69 & 0.33 & 129 & 200 & 0.45 & 0.03 & 0.01 \\
\hline
\end{tabular}

Description of the simulations The laser shock peening simulation has been carried out using the finite element commercial code ABAQUS 6.14. A single impact is considered so an axisymmetric analysis has been performed: the geometrical model used to simulate the laser shock peening consists of a cylindrical cell as shown in Fig. 2a. In order to avoid edge effects, the mesh is divided into two regions. The first one with dimensions $5 \times 5 \mathrm{~mm}$ is meshed with $1 \times 1 \mu \mathrm{m}$ CAX4R elements and the second region is meshed with infinite CINAX4 elements as nonreflecting conditions to avoid shock wave reflections on the free back surface. In terms of boundary conditions, the bottom surface of the sample has been fixed. To obtain stable results, two distinct computation steps were considered: (i) a dynamic explicit analysis is first performed to track the shock wave propagation until the stabilization of the plastic deformation and (ii) an implicit analysis which consists in computing the equilibrium residual stress field due to the induced plastic strains.

The temporal evolution of the applied pressure $P(t)$ is typical of the profiles observed experimentally (Le Bras et al., 2019) and is represented in Fig. 2b. The spatial profile follows a Gaussian shape (see Le Bras et al. (2019)) and the laser spot diameter used in the simulations is $5 \mathrm{~mm}$.

We consider the case of an aluminum alloy (AL000645) investigated by Le Bras et al. (2019) and subjected to a single shock. Laser shock peening involves mechanical wave propagation with very high strain rates, up to $10^{6} \mathrm{~s}^{-1}$. Thus, in addition to an isotropic elastic behavior characterized by the Young's modulus $E$ and the Poisson ratio $v$, an appropriate Johnson-Cook constitutive model accounting for strain rate effects has been considered to describe viscoplasticity (Peyre et al., 2003). The effective yield stress $\sigma_{Y}$ is then given by

$\sigma_{Y}=\left(\sigma_{0}+h p^{n}\right)\left(1+C \log \left(\frac{\dot{\epsilon}^{\mathrm{p}}}{\epsilon_{0}}\right)\right)$,

where $\sigma_{0}$ is the initial yield stress, $h$ the hardening modulus, $n$ the hardening exponent and $p$ the cumulated plastic strain (which are calibrated in quasi-static tensile tests). The strain rate sensitivity is accounted for through the parameters $C$ and $\epsilon_{0}$, which are respectively the strain rate sensitivity parameter and the reference strain rate, $\dot{\epsilon}^{\mathrm{p}}$ being the plastic strain rate during propagation. The material constants used in the numerical simulations have been calibrated in Le Bras et al. (2019) and are given in Table 1.

After convergence of the calculation, we extract the stress profile $\sigma_{11}$ on the top surface of the specimen $\left(x_{3}=0\right)$ which will serve as a reference stress profile for the reconstruction method (see Fig. 2c).

$X$-ray diffraction data treatment Several average datasets $\Sigma_{11}$ are generated from the reference spatial distribution of the residual stress $\sigma_{11}$ computed numerically, in order to simulate X-ray diffraction experiments (based on the psi tilt method) in various situations. The local stress is studied in the interval $[-5,5] \mathrm{mm}$ discretized with $N=101$ points, so the spatial scale is $\Delta x=0.1 \mathrm{~mm}$. Two different collimator sizes representative of laboratory X-ray beams have been considered, a small one corresponding to an irradiated area of width $2 a=2.5 \mathrm{~mm}$ generating 75 "measurement points" and a large one corresponding to an irradiated area of width $2 a=4.5 \mathrm{~mm}$ generating 55 "measurement points". Each dataset is defined in the interval $[-5+a, 5-a]$ because the collimator cannot scan the entire interval due to the irradiated width.

The influence of noise is also studied. Uniformly distributed numbers $\varepsilon_{\sigma}$ (with zero mean) are randomly generated in the interval $\left[-\bar{\varepsilon}_{\sigma}, \bar{\varepsilon}_{\sigma}\right]$ and added to the average stress distribution in order to mimic 
Applied pressure $P(t)$ on a

$5 \mathrm{~mm}$ diameter laser spot

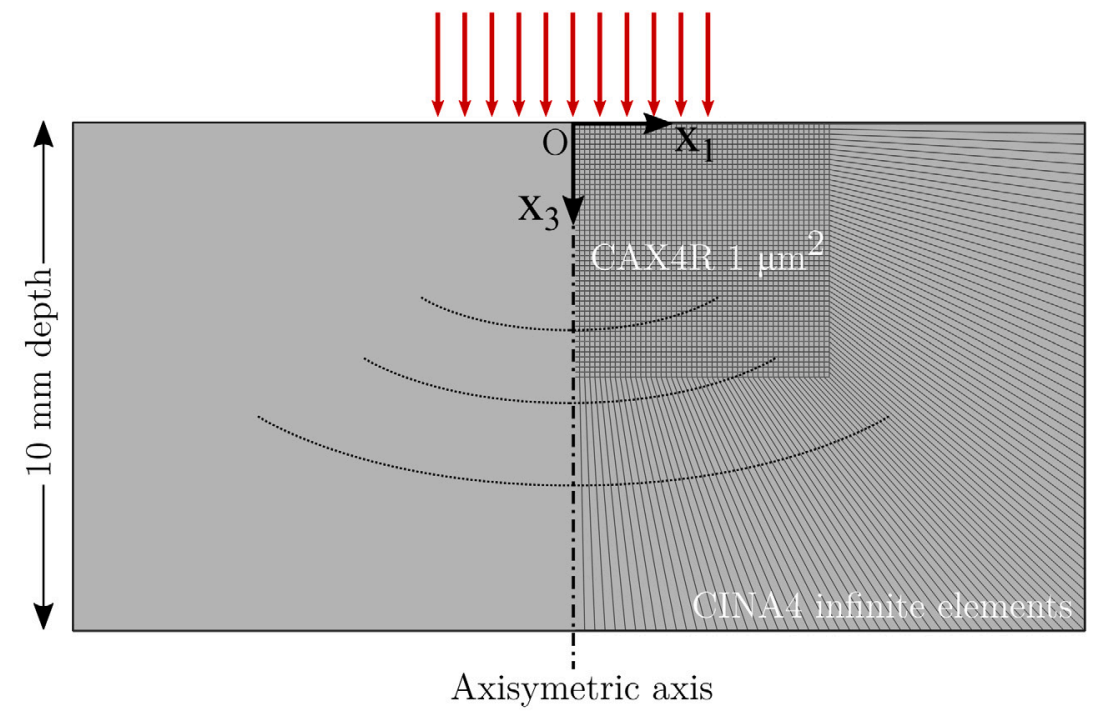

(a)

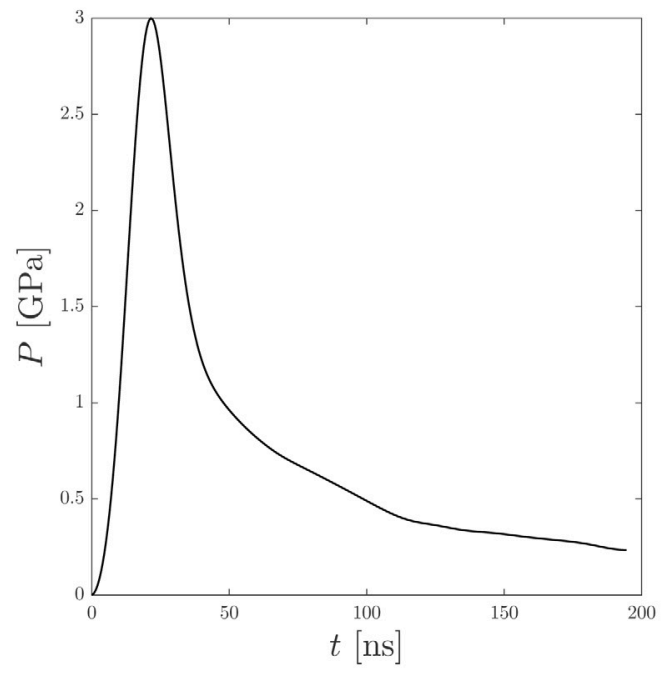

(b)

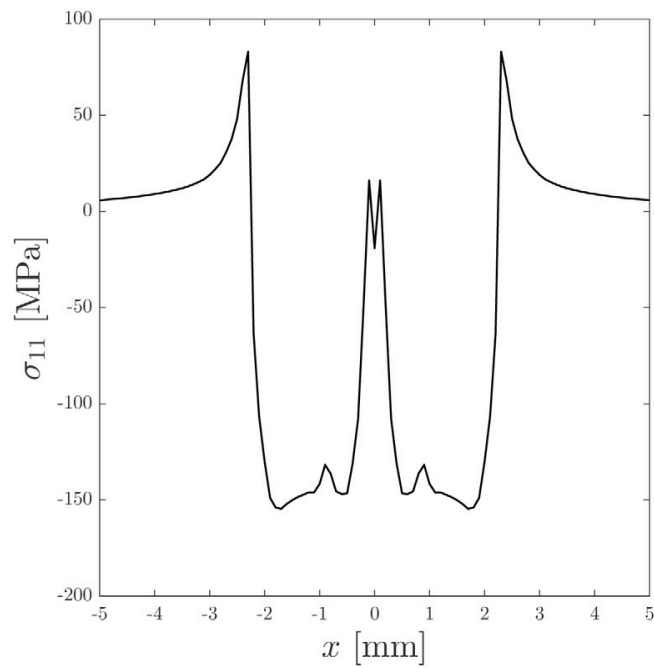

(c)

Fig. 2. Laser shock peening simulation. (a) Geometrical model, (b) Temporal pressure profile $P(t)$, (c) Spatial distribution of the residual stress $\sigma_{11}$.

experimental noise:

$\Sigma_{11}^{\text {noisy }}=\Sigma_{11}+\varepsilon_{\sigma}, \quad \varepsilon_{\sigma} \in\left[-\bar{\varepsilon}_{\sigma}, \bar{\varepsilon}_{\sigma}\right]$.

In the following, the value $\bar{\varepsilon}_{\sigma}=10 \mathrm{MPa}$ will be considered, which is typical of the experimental uncertainties observed in XRD laboratory conditions when aluminum alloys are investigated.

The average datasets for the two collimator sizes are shown in Fig. 3. Without noise, the two average datasets are very different in terms of stress level and inflections points and they both are very far from the exact local field. In particular, both average datasets fail to predict high stress gradients, notably around $x \approx 0 \mathrm{~mm}$ and $x \approx 2 \mathrm{~mm}$. It is worth noting that the addition of noise slightly decreases the difference between the two average datasets, which in that case are quite close.

Reconstruction of the residual stress field Since the two average datasets consist in a total of 130 points, the system given by Eq. (19) can be approximately solved using Eq. (22) in order to reconstruct the local stress (consisting in 101 points). The reconstructed stress profiles are given in Fig. 3 for the two cases considered (with and without noise). In absence of noise in the average datasets, the reconstructed data coincide perfectly with the reference local stress used to generate the average. Very small oscillations are observed close to the interval boundaries which is expected since the datasets contains less information at the boundaries due to the irradiated area. In presence of noise, the reconstructed data are very close to the reference data, except at the boundaries where moderate oscillations are observed. The zones with high stress gradients $(x \approx 0 \mathrm{~mm}$ and $x \approx 2 \mathrm{~mm})$ are quantitatively well reproduced in that case. Moderate discrepancies are observed near the boundaries, but overall the reconstructed data are in very good agreement with the original data, which emphasizes that the deconvolution method can reconstruct a good approximation of the local stress field. 


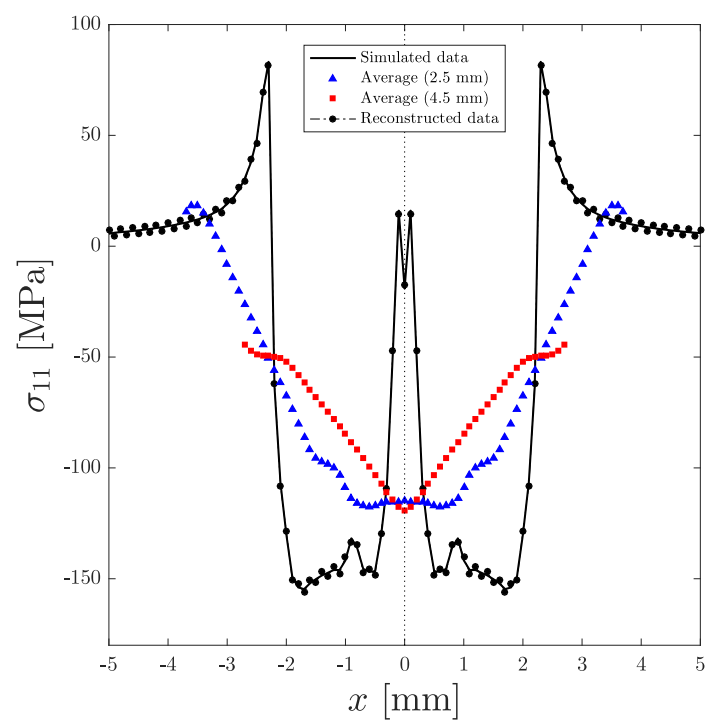

(a)

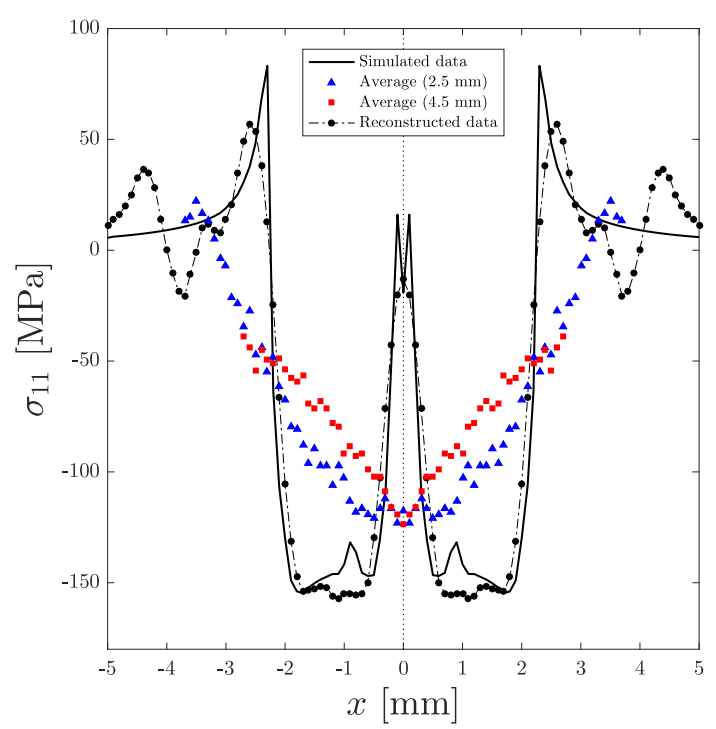

(b)

Fig. 3. Average datasets and reconstruction of the residual stress profile $\sigma_{11}$ is laser shock peening simulation. (a) Without noise and (b) With noise $\left(\bar{\varepsilon}_{\sigma}=10 \mathrm{MPa}\right.$ ).

3.2 Reconstruction of residual stresses in severe plastic deformation processing

We consider now the numerical simulation of residual stresses in heterogeneous severe plastic deformation processing. We focus on repetitive corrugation and straightening (RCS) processing which is repetitive process that allows to achieve grain refinement in sheets (Huang et al., 2004). This process consists of two steps (see Fig. 4a):

(1) An initial flat sheet is corrugated using two corrugation dies, which induces important shear deformation within the sheet. At the end of this step, the specimen is no longer a sheet as its shape is corrugated.

(2) The corrugated specimen is then straightened using two flat straightening dies. At the end of this step, the specimen is again a flat sheet.

These two steps correspond to a pass. Since at the end of a pass the specimen is flat, it is possible to repeat these passes in order to increase the shear deformation, which ultimately leads to some grain refinement (Huang et al., 2004). The presence of highly heterogeneous residual stresses, due to the heterogeneous plastic deformation paths, is an important feature of the process (Elizalde et al., 2020; Ezequiel et al., 2020).

Description of the simulations We consider here the two steps of the first pass of the process, consisting of one corrugation followed by a straightening following the conditions given in Ezequiel et al. (2020). The die profile is based on two perpendicular sinusoidal functions of period $16 \mathrm{~mm}$ (see Elizalde et al. (2020)) which is suitable for a sheet thickness of about $1 \mathrm{~mm}$. Thus, the size of an elementary RCS pattern is $16 \times 16 \times 1 \mathrm{~mm}$. Due to the repetition of corrugation patterns (see Fig. 4a), we can restrict our analysis to the study of one-quarter of a single RCS pattern with symmetric boundary conditions, so the simulation is performed for a specimen of size $8 \times 8 \times 1 \mathrm{~mm}$.

The corrugation dies and straightening plates are supposed to be rigid and the sheet is deformable. The mesh is composed of 5121 linear R3D3 and R3D4 elements for each straightening plate, 7958 R3D4 elements for each corrugation die and 79060 C3D8R elements for the sheet (see Fig. $4 \mathrm{~b}$ ). The average size of an element in the sheet is thus of about $10 \times 10 \times 10 \mu \mathrm{m}$.
Table 2

Material parameters for the 6061 aluminum alloy used for RCS simulation.

\begin{tabular}{llllll}
\hline Material & $E(\mathrm{GPa})$ & $v$ & $\sigma_{0}(\mathrm{MPa})$ & $h(\mathrm{MPa})$ & $n$ \\
\hline AA 6061 & 69 & 0.33 & 160 & 170 & 0.3 \\
\hline
\end{tabular}

We consider the case of a precipitation-hardened aluminum alloy 6061. The behavior is supposed to be rate-independent and follows a classical power-law isotropic hardening. The material constants are given in Table 2. The contact between the dies and the test sample was supposed to follow a Coulomb model. Since in practice the dies are made in steel, a friction coefficient of $\mu=0.3$ was considered, which is typical of the aluminum-steel pair with grease (Ezequiel et al., 2020).

The residual stress $\sigma_{22}$ is extracted on the profile along the line $x_{2}=0$ on the lower surface of the test specimen, which will serve as a reference stress profile for the reconstruction (see Fig. 4c).

$X$-ray diffraction data treatment and reconstruction Several average datasets $\Sigma_{22}$ are generated from the local stress profile $\sigma_{22}$ computed numerically. The local stress is studied in the interval $[-8,8] \mathrm{mm}$ discretized with $N=101$ points, so the spatial scale is $\Delta x=0.16$ $\mathrm{mm}$. For the average calculations, we consider again two collimator sizes, a small one corresponding to an irradiated width of $2 a=2.5 \mathrm{~mm}$ generating 85 "measurement points" and a large one corresponding to $2 a=4.5 \mathrm{~mm}$ generating 73 "measurement points".

Again, additional noise, defined by Eq. (25), was considered in one case with the value $\bar{\varepsilon}_{\sigma}=10 \mathrm{MPa}$. The average datasets for the two collimator sizes are shown in Fig. 5. Without noise, the two average datasets are different, as the averaging effect is more pronounced with the large collimator. In comparison with the exact stress profile, both average datasets fail to reproduce local patterns, notably around $x_{1} \approx 0$ $\mathrm{mm}$ and $x_{1} \approx \pm 5 \mathrm{~mm}$.

Since the two average datasets consist in a total of 158 points, the system given by Eq. (19) can be approximately solved using Eq. (22) in order to reconstruct the local stress (consisting in 101 points). The reconstructed stresses are given in Fig. 5 with and without additional noise. In absence of noise in the average datasets, the reconstructed profile coincides perfectly with the reference local stress used to generate the average. In presence of noise, the reconstructed profile is also very close to the reference profile. The original features of the initial profile that were erased by the average (around $x_{1} \approx 0 \mathrm{~mm}$ and $x_{1} \approx \pm 5$ 


\section{RCS Step 1: Corrugation}

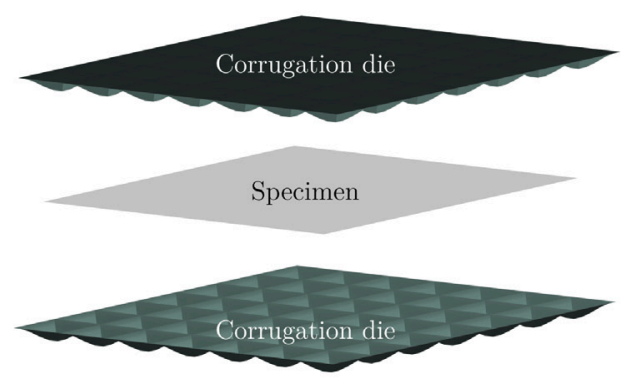

\section{RCS Step 2: Straightening}



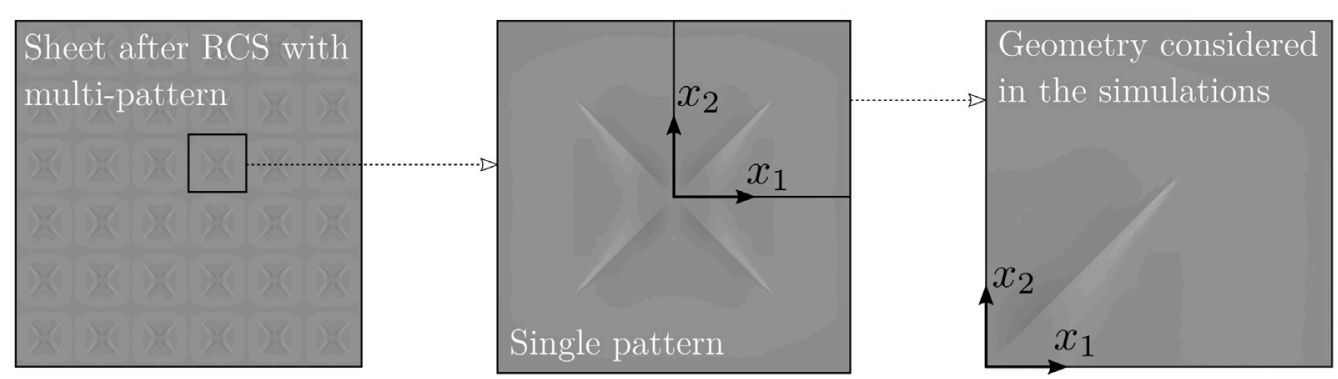

(a)



(b)



(c)

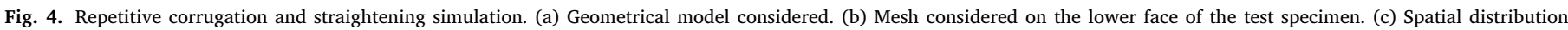
of the residual stress $\sigma_{22}$ on the line $x_{2}=0$.

$\mathrm{mm}$ ) are perfectly reproduced by the reconstruction method. Again, it should be noted that some discrepancies are observed near boundaries which is expected since there is less information for the reconstruction due to the irradiated area which cannot exceed the specimen domain.

\section{Application of the method to experimental data}

The deconvolution method is now applied to experimental data collected on a specimen processed by repetitive corrugation and straightening processing.

\subsection{Description of the $X$-ray diffraction experiments}

A precipitation-hardened aluminum alloy 6061 was considered. A solid solution heat treatment at $803 \mathrm{~K}$ for two hours was applied, then the sample was water-quenched, followed by an aging treatment at $453 \mathrm{~K}$ for $18 \mathrm{~h}$ (Elizalde et al., 2020). The chemical composition of the Al-6061 alloy is given in Table 3.

The AA-6061 sheets were processed by heterogeneous RCS at room temperature with a geometrical 2D corrugation die based on two 




(a)

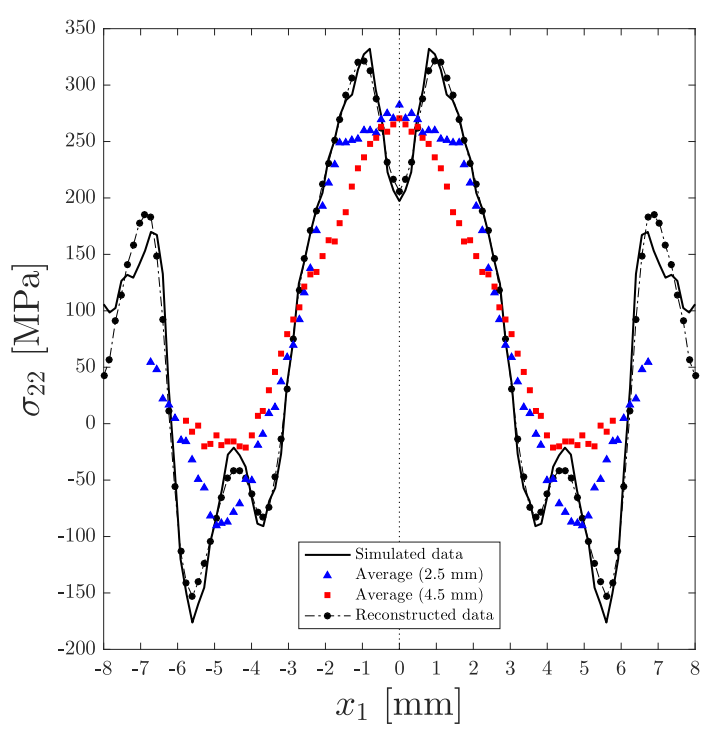

(b)

Fig. 5. Average datasets and reconstruction of the residual stress $\sigma_{22}$ is RCS simulation. (a) Without noise and (b) With noise $\left(\bar{\varepsilon}_{\sigma}=10\right.$ MPa).



Specimen after corrugation

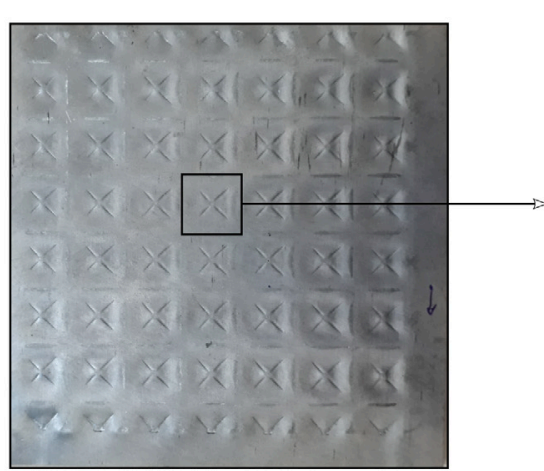

Specimen after straightening

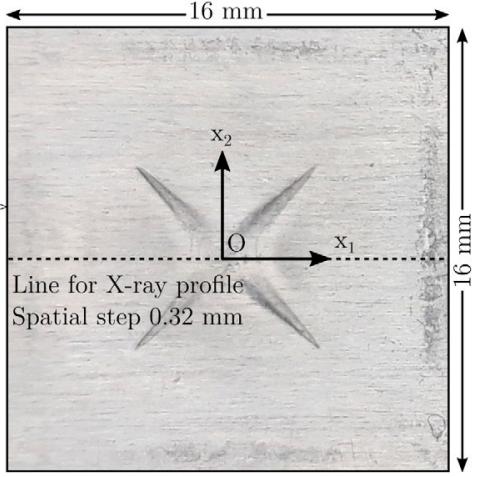

Elementary pattern considered for X-ray measurements

Fig. 6. Description of the specimen processed by a one pass RCS.

Table 3

Composition of the Al-6061 alloy.

\begin{tabular}{llllllllll}
\hline Element & $\mathrm{Si}$ & $\mathrm{Fe}$ & $\mathrm{Cu}$ & $\mathrm{Mn}$ & $\mathrm{Mg}$ & $\mathrm{Cr}$ & $\mathrm{Zn}$ & $\mathrm{Ti}$ & $\mathrm{Al}$ \\
\hline wt $\%$ & 0.77 & 0.24 & 0.16 & 0.03 & 1.03 & 0.07 & 0.03 & 0.02 & Balance
\end{tabular}

Table 4

Experimental conditions of X-ray diffraction.

\begin{tabular}{llllll}
\hline $\begin{array}{l}\mathrm{Cr}-\mathrm{K} \alpha \\
\text { radiation }\end{array}$ & Voltage & Current & $\begin{array}{l}\mathrm{XRD} \\
\text { planes }\end{array}$ & $\begin{array}{l}\text { Angle } \\
2 \theta\end{array}$ & $\Psi$ angles \\
\hline $0.2290 \mathrm{~nm}$ & $20 \mathrm{kV}$ & $1 \mathrm{~mA}$ & $\left(\begin{array}{lll}3 & 1 & 1\end{array}\right)$ & $139^{\circ}$ & 13 angles in $\left[-37.27^{\circ}, 39.23^{\circ}\right]$ \\
\hline
\end{tabular}

orthogonal sinusoidal profiles ${ }^{1}$ as presented in Section 3.2. The die is composed of $7 \times 7$ elementary patterns of size $16 \times 16 \mathrm{~mm}$ and the size of the processed sheet was about $120 \times 120 \times 1 \mathrm{~mm}$ (see Fig. 6). The RCS process was carried out using a hydraulic press Mecamaq DE-80 at a maximum pressure of 10 tons. As in Section 3.2, only one pass is

1 The comprehensive details related to the design and machining of the sinusoidal RCS dies can be found in Elizalde et al. (2020). considered, that is a corrugation of an initial flat sheet followed by a straightening (see Fig. 6).

The residual stress distribution $\sigma_{22}$ was evaluated at the bottom surface of a one pass corrugated and straightened specimen by $\mathrm{X}$ ray diffraction measurements using the psi tilt method and an in-situ diffractometer type X-RAYBOT (manufactured by MRX France). The XRD measurements were performed along the line $x_{2}=0$ (see Fig. 6) with a spatial step of $0.32 \mathrm{~mm}$. Two collimators were considered, a small one with a circular irradiated area with a diameter of $2.5 \mathrm{~mm}$, and a large one with a circular irradiated area with a diameter of $4.5 \mathrm{~mm}$, which permits to provide two average datasets. The X-ray diffraction conditions are given in Table 4. In the conditions of this study, the measurement uncertainties are of about $\pm 10 \mathrm{MPa}$.

\subsection{Results}

The two experimental average datasets are shown in Fig. 7. They consist in 43 measurement points for the small collimator and 37 measurements points for the large collimator. Since the die geometry is symmetric with respect to the $x_{2}$-axis, the experimental data have been post-treated in order to enforce symmetry of the datasets with respect to the $x_{2}$-axis, which allows to reduce the experimental uncertainties. 
The automatic smoothing of the experimental data are represented in Fig. 7 by continuous lines. The effect of collimator size is very important in terms of inflection points and stress levels. A maximum difference of about $100 \mathrm{MPa}$ is notably observed near the center of the specimen. The stress gradients associated with the large collimator are smaller than that associated with the small collimator, which confirms that a strong averaging effect is induced by the measure.

The local stress to be reconstructed is looked for in the interval $[-8,8] \mathrm{mm}$ discretized with the same spatial scale than the experimental results, that is $\Delta x=0.32 \mathrm{~mm}$. Thus the number of points for the local reconstructed stress is $N=51$, which is lower than the 80 experimental measurements points, so the system (19) can thus be approximately solved using Eq. (22). The reconstructed stress profile is represented in Fig. 7.

The stress profile reconstructed is considerably different from the average datasets collected experimentally. High stress gradients and inflection points are predicted near $x_{1} \approx 0 \mathrm{~mm}$ and $x_{1} \approx 4 \mathrm{~mm}$, in contrast with the average datasets. It is worth noting that the reconstructed stress profile as well as the average datasets are very similar to the local stress simulated numerically in Section 3.2 in similar conditions. In particular, the shape profile of the local stress corresponds qualitatively to that simulated numerically, with the same exact location of inflection points.

\section{Discussion}

The experimental results collected on a specimen processed by a single pass RCS have highlighted the strong averaging effect observed on residual stresses which is related to the irradiated area in X-ray diffraction measurements. This confirms that X-ray diffraction results must be carefully interpreted (Hennion et al., 2000), in particular in cases where heterogeneous strains are involved such as in severe plastic deformation processing. This suggests that a systematic analysis of the influence of the irradiated area size is needed to assess the possible averaging effect of the measure.

The deconvolution method developed in Section 2 has permitted to reconstruct a unique, supposedly local, residual stress field from two average datasets. This reconstruction requires a large number of measurements which can be easily performed by an automated or robotized spectrometer, allowing a precise mapping of the specimen surface. The method is based on experimental data which have necessarily some uncertainty. The presence of noise will thus deteriorate the reconstructed profile as shown in Section 3, since it is based on some approximate smoothed stress averages. Indeed, although an automatic smoother used, the smoothed averages will necessarily differ from the exact ones, inducing errors in the reconstruction. It must be noted that with a reasonable value for the experimental noise, this effect is quite small and a very good agreement was observed between the reconstructed and the reference stress profiles. Thus the quality of the reconstructed is not guaranteed but it contains more information than the average datasets and appears to be a more relevant quantity for the comparison with the local results of full-field finite element simulations.

In the case of RCS, the corrected stress field has the same exact profile than that calculated numerically in the same processing conditions, which suggests that the deconvolution method has captured the essential features of the stress profile. Some slight differences are observed between the numerical and reconstructed stress profiles, which can be due to several factors:

(1) The numerical simulation of RCS has been performed with several arbitrary (but reasonable) values of parameters (as the friction coefficient and the applied forces of straightening plates) which are difficult to measure experimentally; the numerical conditions RCS processing may slightly differ from the experimental ones. No attempt was made in the numerical simulations to adjust the material and process parameters, which explains

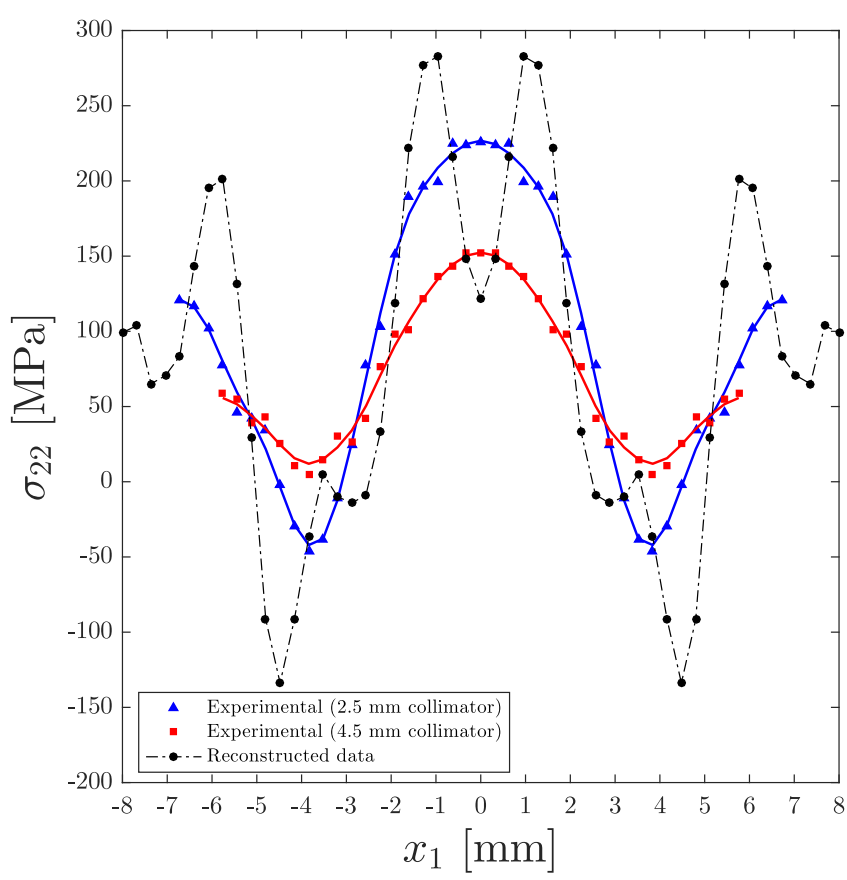

Fig. 7. Average datasets and reconstruction of the residual stress $\sigma_{22}$ in RCS processing.

that the stress profile obtained numerically does not coincide perfectly with that reconstructed.

(2) The reconstruction method was derived under some simplifying assumptions that could induce some discrepancies:

- We assumed that the stress gradient effects only occur in one direction of the space in order to derive a model that requires experimentally a mapping in one direction. In practice, this hypothesis can be questionable because gradients can occur in two directions and the irradiated area is circular; the average effect should be considered in a two-dimensional area. In the problem considered (RCS), the stress distribution calculated numerically suggests that, for the profile considered $\left(x_{2}=0\right)$, the $2 \mathrm{D}$ gradient effect is negligible in most of the specimen. Thus in the case treated, if the reconstructed stress should not be greatly affected by the $2 \mathrm{D}$ effects, some (small) discrepancies may be induced by the $2 \mathrm{D}$ averaging effect.

- We did not take into account the penumbra effect which corresponds to a non-uniform intensity distribution inside the irradiated area related to (i) the incident-beam inclination and (ii) the collimator size. It has been shown in Hennion et al. (2000) that this effect is generally negligible when "large" collimators are used, typically with size giving an irradiated area larger than $1 \mathrm{~mm}$, which is the case in this work. If small collimators are used, it would be necessary to modify the definition of the average stress by considering some weight according to the irradiated area size.

- The accuracy of the deconvolution method depends on the size of the overdetermined system (19) and on the spatial discretization. A decrease of the spatial scale is thus expected to allow a better representation of local stress gradients. Moreover, an increase of the number of datasets, through the use of multiple collimator sizes, is also expected to increase to quality of the reconstructed residual stress field. 
It should also be noted that in presence of highly heterogeneous strain of order of the material grain size, the agreement between the reconstructed stress and finite element simulations could be improved by including appropriate heterogeneous and anisotropic material properties in the simulations (Wang et al., 2008).

\section{Conclusion}

The aim of this work was to develop a spatial deconvolution technique in order to reconstruct the heterogeneous residual stress profiles from diffraction data.

First, a method of reconstruction was developed based on the inversion of a linear system constructed using average datasets that are obtained with several collimators of different diameters. The method was first applied to the reconstruction of heterogeneous residual stress profiles in several reference cases (laser shock peening and repetitive corrugation and straightening processing) where the surface stress distributions are determined numerically by the finite element method; the average datasets were calculated from the reference stress profile with additional noise in order to mimic measurement uncertainties. In both processes considered, a very good agreement is observed between the reference stress profiles and the ones reconstructed even with a high level of noise. The method was finally applied to experimental Xray diffraction data on a specimen processed by repetitive corrugation and straightening in similar conditions as the numerical simulations. A strong averaging effect was observed on the experimental results and a good agreement was observed between the local stress profile reconstructed and that predicted numerically.

The present paper shows that the residual stress profiles determined by X-ray diffraction can be far from the exact local stress field in presence of high surface stress gradients due to the irradiated area. It explains the gaps that are generally observed between experimental residual stress profiles and the results of numerical simulations, which are (often) wrongly attributed to a poor calibration of model parameters in finite element simulations. The deconvolution technique proposed in this study allows a better reconstruction of local stress profiles which should improve the comparison between experimental results and numerical calculations.

\section{Declaration of competing interest}

The authors declare that they have no known competing financial interests or personal relationships that could have appeared to influence the work reported in this paper.

\section{References}

Baczmanski, A., Braham, C., Seiler, W., Shiraki, N., 2004. Multi-reflection method and grazing incidence geometry used for stress measurement by X-ray diffraction. Surf. Coat. Technol. 182, 43-54.

Ben Fredj, N., Ben Nasr, M., Ben Rhouma, A., Sidhom, H., Braham, C., 2004. Fatigue life improvements of the AISI 304 stainless steel ground surfaces by wire brushing. J. Mater. Eng. Perform. 13, 564-574.

Ben Rhouma, A., Sidhom, N., Makhlouf, K., Sidhom, H., Braham, C., Gonzalez, G., 2019. Effect of machining processes on the residual stress distribution heterogeneities and their consequences on the stress corrosion cracking resistance of AISI 3161 SS in chloride medium. Int. J. Adv. Manuf. Technol. 105, 1699-1711.

Brockman, R.A., Braisted, W.R., Olson, S.E., Tenaglia, R.D., Clauer, A.H., Langer, K., Shepard, M.J., 2012. Prediction and characterization of residual stresses from laser shock peening. Int. J. Fatigue 36, 96-108.

Craven, P., Wahba, G., 1978. Smoothing noisy data with spline functions. Numer. Math. 31, 377-403.

Dong, P., Brust, F.W., 2000. Welding residual stresses and effects on fracture in pressure vessel and piping components: A millennium review and beyond. J. Press. Vessel Technol. 122, 329-338.

Elizalde, S., Ezequiel, M., Figueroa, I.A., Cabrera, J.M., Braham, C., Gonzalez, G., 2020. Microstructural evolution and mechanical behavior of an al-6061 alloy processed by repetitive corrugation and straightening. Metals 10 (489).

Engl, H.W., Hanke, M., Neubauer, A., 1996. Regularization of Inverse Problems. Springer Science \& Business Media.
Ezequiel, M., Figueroa, I.A., Elizalde, S., Cabrera, J.M., Braham, C., Morin, L., Gonzalez, G., 2020. Numerical and experimental study of a 5754-aluminum alloy processed by heterogeneous repetitive corrugation and straightening. J. Mater. Res. Technol. 9, 1941-1947.

Fang, Z.C., Wu, Z.L., Huang, C.G., Wu, C.W., 2020. Review on residual stress in selective laser melting additive manufacturing of alloy parts. Opt. Laser Technol. 129, 106283.

Garcia, D., 2010. Robust smoothing of gridded data in one and higher dimensions with missing values. Comput. Statist. Data Anal. 54, 1167-1178.

Garcia, D., 2011. A fast all-in-one method for automated post-processing of PIV data. Exp. Fluids 50, 1247-1259.

Hammond, D.W., Meguid, S.A., 1990. Crack propagation in the presence of shot-peening residual stresses. Eng. Fract. Mech. 37, 373-387.

Hennion, V., Sprauel, J.M., Michaud, H., 2000. Contribution to residual-stress evaluation in high-stress-gradient zones by X-ray diffraction. J. Appl. Crystallogr. 33, 26-34.

Huang, J., Zhu, Y.T., Alexander, D.J., Liao, X., Lowe, T.C., Asaro, R.J., 2004 Development of repetitive corrugation and straightening. Mater. Sci. Eng. A 371, 35-39.

Jacobus, K., DeVor, R.E., Kapoor, S.G., 2000. Machining-induced residual stress: Experimentation and modeling. J. Manuf. Sci. Eng. 122, 20-31.

Kahloun, C., Badawi, P., Viaris de Lesegno, P., 1994. X-ray Analysis. The case of substantial stress gradients and strong heterogeneity. In: Proceedings of the 4th International Conference on Residual Stresses, Baltimore, Maryland, USA.

Kahloun, C., Badji, R., Queyreau, S., Franciosi, P., Bacroix, B., 2014. Spatial convolution of a stress field analyzed by X-ray diffraction. Adv. Mater. Res. 996, 169-174.

Le Bras, C., Rondepierre, A., Seddik, R., Scius-Bertrand, M., Rouchausse, Y., Videau, L., Fayolle, B., Gervais, M., Morin, L., Valadon, S., Ecault, R., Furfari, D., Berthe, L., 2019. Laser shock peening: Toward the use of pliable solid polymers for confinement. Metals 9, 793 .

Leggatt, R.H., 2008. Residual stresses in welded structures. Int. J. Press. Vessels Pip. 85, 144-151.

Lu, J., 1996. Handbook of Measurement of Residual Stresses. Fairmont Press.

Lu, G., Trdan, U., Zhang, Y., Dulaney, J.L., 2020. The distribution regularity of residual stress on a metal surface after laser shock marking. Mech. Mater. 143, 103310.

Mahmoudi, A.H., Ghasemi, A., Farrahi, G.H., Sherafatnia, K., 2016. A comprehensive experimental and numerical study on redistribution of residual stresses by shot peening. Mater. Des. 90, 478-487.

Marciszko, M., Baczmański, A., Braham, C., Wróbel, M., Wroński, S., Cios, G., 2017. Stress measurements by multi-reflection grazing-incidence X-ray diffraction method (MGIXD) using different radiation wavelengths and different incident angles. Acta Mater. 123, 157-166.

Montross, C.S., Wei, T., Ye, L., Clark, G., Mai, Y.W., 2002. Laser shock processing and its effects on microstructure and properties of metal alloys: a review. Int. J. Fatigue 24, 1021-1036.

Peyre, P., Berthe, L., Vignal, V., Popa, I., Baudin, T., 2012. Analysis of laser shock waves and resulting surface deformations in an al-cu-li aluminum alloy. J. Phys. D: Appl. Phys. 45, 335304.

Peyre, P., Fabbro, R., 1995. Laser shock processing: a review of the physics and applications. Opt. Quantum Electron. 27, 1213-1229.

Peyre, P., Sollier, A., Chaieb, I., Berthe, L., Bartnicki, E., Braham, C., Fabbro, R., 2003. FEM Simulation of residual stresses induced by laser peening. Eur. Phys. J. - Appl. Phys. 23, 83-88.

Reyes-Ruiz, C., Figueroa, I.A., Braham, C., Cabrera, J.M., Zanellato, O., Baiz, S., Gonzalez, G., 2016. Residual stress distribution of a 6061-t6 aluminum alloy under shear deformation. Mater. Sci. Eng. A 670, 227-232.

Rossini, N.S., Dassisti, M., Benyounis, K.Y., Olabi, A.G., 2012. Methods of measuring residual stresses in components. Mater. Des. 35, 572-588.

Vaara, J., Kunnari, A., Frondelius, T., 2020. Literature review of fatigue assessment methods in residual stressed state. Eng. Fail. Anal. 110, 104379.

Wang, Y., Kysar, J.W., Yao, Y.L., 2008. Analytical solution of anisotropic plastic deformation induced by micro-scale laser shock peening. Mech. Mater. 40, 100-114.

Webster, G.A., Ezeilo, A.N., 2001. Residual stress distributions and their influence on fatigue lifetimes. Int. J. Fatigue 23, 375-383.

Xie, L., Wang, C., Wang, L., Wang, Z., Jiang, C., Lu, W., Ji, V., 2016. Numerical analysis and experimental validation on residual stress distribution of titanium matrix composite after shot peening treatment. Mech. Mater. 99, 2-8.

Zhang, W., Fang, K., Hu, Y., Wang, S., Wang, X., 2016. Effect of machining-induced surface residual stress on initiation of stress corrosion cracking in 316 austenitic stainless steel. Corros. Sci. 108, 173-184.

Zhou, J., Sun, Z., Kanouté, P., Retraint, D., 2018. Reconstruction of residual stress and work hardening and their effects on the mechanical behaviour of a shot peened structure. Mech. Mater. 127, 100-111. 